\title{
Atmospheric Corrosion of Stainless Steel 304
}

\author{
Fakher Jribi $^{1}$, Christiaan Adika Adenya ${ }^{2}$ and James Mutuku Mutua ${ }^{3}$ \\ ${ }^{1}$ Department of Mechanical engineering, Pan African University, PAUSTI, Kenya. \\ ${ }^{2}$ Department of Marine Engineering and Maritime operations, JKUAT. \\ ${ }^{3}$ Department of Mechanical engineering, JKUAT. \\ ${ }^{1}$ ORCID: 0000-0001-9801-3421(Fakher Jribi)
}

\begin{abstract}
Metal Materials are used for the construction of structures in sub-Saharan Africa. The typical welding and joining techniques are the Metal Inert Gas, Tungsten Inert Gas and Arc welding. Atmospheric corrosion is a serious problem and if not properly maintained, they can quickly become liabilities because of corrosion-generated leaks and service interruptions. Although the study of the corrosion are often simplified, the impact of the tanks for maintaining an adequate quality of water in the network without corrosion is of paramount. Three samples were exposed for nine days to outdoors atmospheric conditions ( $\mathrm{C} 3$ corrosivity) and then to an immersion test for 30 days equivalent to 120 cycles according to the ASTM G50 and ASTM G44 standards. The weight-loss method was used for the measurements of weight loss of the material as a function of time. The samples were cut and welded using arc welding, metal inert gas and tungsten inert gaz. The welded samples were compared, and the result of the corrosion rate performed. The corresponding rate of corrosion from experiment for arc welding and Metal Inert Gas was $0.01 \mathrm{~mm} / \mathrm{y}$ and for Tungsten Inert Gas was $0.004 \mathrm{~mm} / \mathrm{y}$. The corrosion resistance of stainless steel was explained by the very thin passive layer that prevents the iron from corrosion. The formation of this passive layer is closely related to the chromium content of the steel with a minimum of $10.5 \%$ Chromium produces a thin layer of oxide on the surface of the steel. Increasing the amount of Chromium gives an increased resistance to corrosion. Metal alloys undergo uniform chemical corrosion that attacks surface evenly. Therefore, the loss of mass per unit area and per unit of time can be determined during the immersion test., For each of the three samples, measurement of the weight loss was done after 40, 80 and 120 cycles for a period of 30 days. The result of this study showed that the arc weld and the metal inert gas corroded faster and has a higher corrosion rate on the weld zone, whereas the tungsten welding was found to be the most resistant against corrosion attack.
\end{abstract}

Keywords: Welding; corrosion; atmospheric corrosion; weight-loss method; rate of corrosion

\section{INTRODUCTION}

For a long time, corrosion has been a problem in sub-Saharan Africa. Corrosion causes the failure of water tanks and other structures that lead to losses. The atmosphere is a significant factor that causes corrosion in Africa. The high humidity, which is above $70 \%$ in sub-Saharan Africa and rainfall contribute to atmospheric corrosion[1]. According to ISO 9223[2], most African countries' atmosphere falls in between moderate (C3) Low-carbon steel Thickness loss $(\mu \mathrm{m})>25$ to 50 include Urban areas and a few coastal areas with low effect from the sea. Subtropical and tropical zones with low pollution in the atmosphere. to severe (C4) with Low-carbon steel Thickness loss $(\mu \mathrm{m})>50$ to 80 . In Contaminated urban areas, industrial areas, certain coastal areas, areas exposed to strong de-icing salts. Subtropical and tropical zone, atmosphere with medium pollution.. For instance, a study of corrosion in Mauritius showed that the state receives an average of $2000 \mathrm{~mm}$ per year of rainfall that leads to high humidity in the country[3].

Metal materials are commonly used in fabricating water tanks in Sub Saharan Africa. Fusion welding methods that include Arc welding, tungsten inert gas (TIG), and metal inert gas (MIG), are the most used welding methods[4]. This research compares corrosion rate for stainless steel joined using the aforementioned methods of welding. A statistic survey by University of Nairobi of 20 companies showed that most companies use stainless steel, mild steel, galvanized steel and aluminum for their fabricated structures and structures made of mild steel were profoundly affected by atmospheric corrosion[5].

304 stainless steel (SS) is one of the most consumable materials were considered to have, in the annealed condition, very good resistance to general and localized corrosion due to their chromium content, which is higher or equal to a critical value around atomic $13 \%$. The exposure of welded metal materials to the environment was the primary cause of corrosion. Severe corrosion was observed in the heat-affected zones compared to other parts of the sample. In the heat-affected zones, increased stress presents another cause of corrosion[6].

The findings of the study will provide data on corrosion rates that will be used to recommend the best welding method to be used and the prevention methods to avoid corrosion.

\section{METHODOLOGY}

\section{A. INTRODUCTION}

The rates of corrosion were inspected using the following:

- Atmospheric exposure of the welded metal materials for nine days.

- Immersion tests (accelerated corrosion reaction) for 30 days. 
According to BS 4360 43D [7], MIG, TIG, and Arc welding, were used to manufacture three welded specimens. The corrosion rates were examined after the atmospheric exposure and the immersion test using the weight loss method and microstructure examination.

\section{B. Short term outdoor atmospheric tests}

The three samples were machined to $150 \mathrm{~mm} \times 50 \mathrm{~mm} \times 5 \mathrm{~mm}$. In welding the samples together, a butt joint was used according to ISO 9692[8] According to ASTM G1-03[9].

The samples were exposed to outdoor in C3 atmosphere, according to ISO EN BS 8565[10].

Details about materials and welding electrodes used are summarized in Table 1 (a).

Table 1(a): Typical chemical composition of the material and electrodes used

\begin{tabular}{|c|c|c|c|c|c|}
\hline \multicolumn{7}{|c|}{ Chemical composition, \% } \\
\hline Material/Electrode & $\mathrm{C}$ & $\mathrm{Si}$ & $\mathrm{Mn}$ & $\mathrm{S}$ & $\mathrm{P}$ \\
\hline BS 4063 & 0.16 & $0.10-0.50$ & 1.50 & 0.040 & 0.040 \\
\hline ER 70s-6 & $0.06-0.15$ & $0.80-1.15$ & $1.4-1.85$ & 0.035 & 0.025 \\
\hline E 6013 & 0.08 & 0.18 & 0.45 & 0.012 & 0.014 \\
\hline
\end{tabular}

Table 1(b): Welding parameters

\begin{tabular}{|c|c|c|c|c|c|}
\hline & Polarity & Current & Voltage & $\begin{array}{c}\text { Electrode/Filler } \\
\text { Size }\end{array}$ & $\begin{array}{c}\text { Gas flow } \\
\text { rate }\end{array}$ \\
\hline MMA & Deep & $100 \mathrm{~A}$ & $17 \mathrm{~V}$ & $2.4 \mathrm{~mm}$ & - \\
\hline MIG & Deep & $160 \mathrm{~A}$ & $19 \mathrm{~V}$ & $0.8 \mathrm{~mm}$ & $15 \mathrm{~L} / \mathrm{min}$ \\
\hline TIG & Deep & $125 \mathrm{~A}$ & - & $2.4 \mathrm{~mm}$ & $10 \mathrm{~L} / \mathrm{min}$ \\
\hline
\end{tabular}

All the welding procedures conform to the specification of ISO 15614-1[11]. To avoid welding defects that can lead to corrosion, the welding set up and parameters were chosen to ensure perfect weld joints. In the process of welding, each welding types had different settings[11]. The electrode used for the MMA welding process was E6013 with $2.5 \mathrm{~mm}$ diameter. For MIG and TIG welding, filler rods used were ER70s-6 with 0.8 and 2.4 diameters respectively.

Fig 1 below shows the stainless steel specimens after outdoor exposure for a period of 30 days:

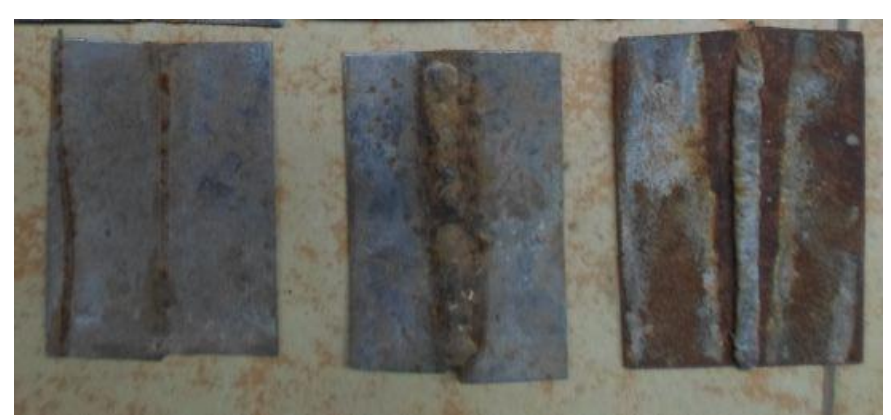

Fig 1: Exposed specimens of stainless steel
After 10 days of exposures, the amount of surface degradation on the samples was analyzed using the ImageJ software that supports standard image processing functions and image analysis.

\section{Alternate immersion tests}

The immersion test is an accelerated corrosion reaction for the three samples which consists of dry and wet procedures. For the outdoor exposure, the stainless steel samples were prepared according to ASTM G1[10]. The Immersion test was carried out in 30 days to investigate the rate of corrosion and the degradation on the materials and welds joints according to ASTM G44[8]. For the immersion test, the minimum volume of distilled water required was $58 \mathrm{~L}$ with conductivity of 3 $\mu \mathrm{S} / \mathrm{cm}$ according to ASTM G44[8], $5 \%$ of salt (sodium chloride) was used for accelerating the reaction, the $\mathrm{PH}$ of the solution is 7 . The alternate wet and dry immersion test in the solution includes $10 \mathrm{~min}$ immersion in the basin followed by 50 min drying in the air. The details of the experimental test are as follows:

- A basin filled with $58 \mathrm{~L}$ of distilled water with $5 \%$ of sodium chloride, a line was drawn to check the losses of water caused by the evaporation, The water level is checked and replenished during the test.

- The humidity in Nairobi-Kenya was found $60 \%$, and the temperature ranges from $23^{\circ} \mathrm{C}$ to $27^{\circ} \mathrm{C}$

- A PH meter was used throughout the immersion test to check the solution $\mathrm{PH}$

The experiment on the samples shows that the atmospheric corrosion C 3 achieved by 120 cycles dry/wet for an equivalent period of 30 days of immersion test. On the other hand, and according to ASTM G1-03[10], the welded samples by TIG, MIG, and arc were removed after every 40 cycles to get the weight loss of each one of them.

Previous calibration of the equipment, using stainless steel samples, showed that $\mathrm{C} 3$ category corrosivity can be achieved by performing the test between 100 to 120 cycles. Hence for the alternate immersion tests, specimens were exposed in sets of three. They were removed after 40, 80, 120 cycles and their weight loss determined, according to ASTM G1-03[10].

\section{RESULTS AND DISCUSSION}

After 40 cycles of exposure, for the MIG welded specimens, it was clearly discerned that the weld area corroded more quickly than the base metal. The weld area suffered from localized corrosion because it was less noble than the base metal. This is known Preferential Weld Corrosion (PWC). Also, the weld suffered accelerated corrosion because of unfavorable area ratio of small weld metal (anodic) and large base metal (cathodic). For the TIG welded specimens, no corrosion products were found in the weld area. The weld surface remained nearly unchanged, whereas the base metal had corroded. After 80 cycles coorosion product were found along the weld zone and slightly in the edge of the samples 
After 120 cycles of exposure, it was observed that the specimens' surface was almost covered with corrosion products. Moreover, it was found that for the MIG welded specimens, the weld had rusted more than the base metal.

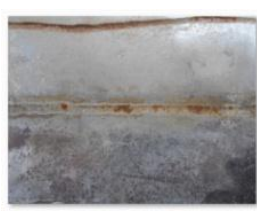

TIG

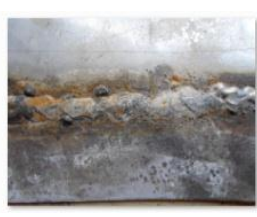

ARC

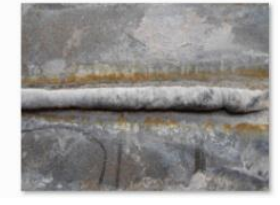

MIG
Fig. 2: Surface of test specimen after 40 cycles of exposure

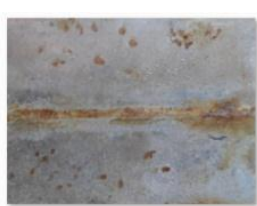

TIG

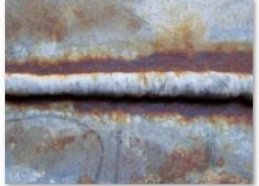

MIG

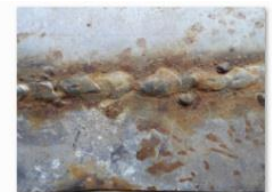

ARC
Fig. 3: Surface of the test specimen after 120 cycles of exposure

To compare the corroded area ratio of the weld metal, corroded area ratio against exposure time (days) for all the 3 specimens were plotted on the graph

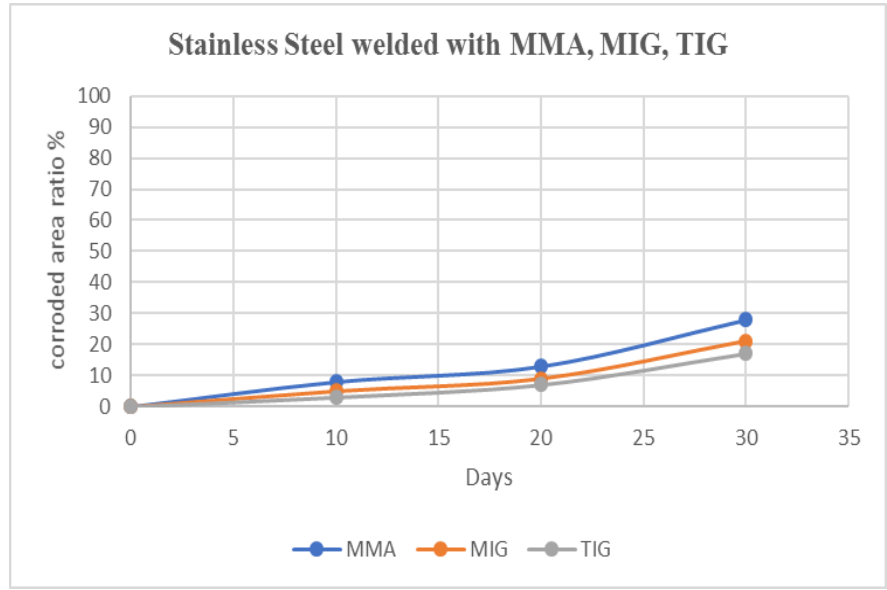

Fig 4: Corroded area ratio against exposure time (days) for Stainless steel

Corroded area ratio of the stainless steel plates welded with MMA were greater than the rates experienced by MIG and TIG specimens, Average corroded area ratio per each surface on these plates extended to above $28 \%$ at 120 cycles, as compared to the less than $21 \%$ and $17 \%$ of average corroded area ratio experienced by MIG and TIG respectively. The corrosion loss data for these base plate configurations appear to be increasing with increased testing cycles.

\section{ACCELERATED CORROSION TESTING}

The alternate immersion was applied to evaluate the atmospheric corrosion resistance. Since the surface can be analysed only for a very short term during atmospheric exposures, the alternate immersion tests were performed to analyze the degradation of the welded joints over an equivalent period of 30 days.

In order to investigate the corrosion behavior of stainless-steel Grade 304, for water tank-specific applications, an appropriate corrosion testing procedure was selected and executed. The chosen corrosion study method ought to produce corrosion products and results representative of those expected in the field, be able to be completed in a reasonable amount of time and be generally accepted by the industries. While in-field studies provide the most accurate representation of realistic corrosive environments, the limited duration of time available for this research project prohibited an in-field corrosion study. As a result, it was decided that an accelerated corrosion study would be implemented.

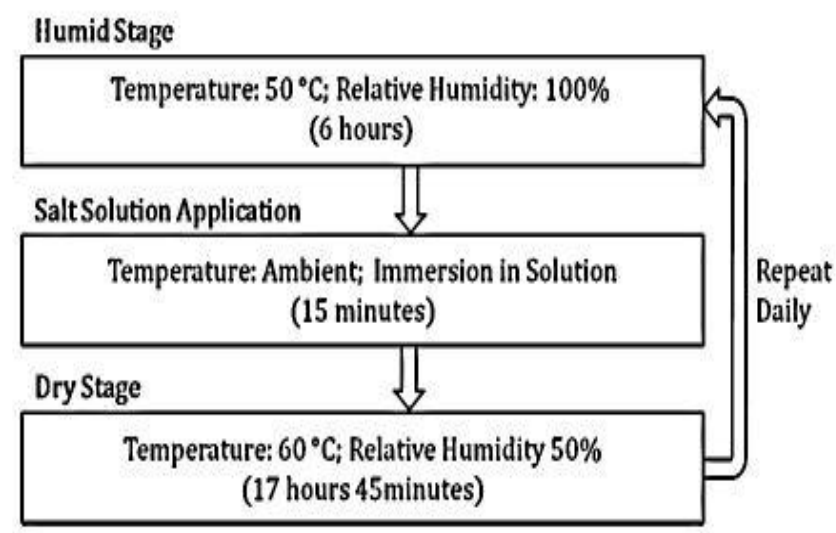

Figure 5: Cyclic testing procedure

In order to fulfill the requirements of the testing procedure described by the modified ASTM G44[11] cyclic corrosion testing procedure, soaking containers were used for the salt application stage. For all stages of testing, non-corrosive equipment was used whenever possible in the handling and positioning of specimens in order to avoid the breakdown of these materials or contamination of the corrosion specimens.

In order to properly orient the specimens during the testing procedure and facilitate an efficient means of transitioning from the environmental to soaking containers, a combination of non-corrosive plastic equipment was used. Particularly, testing procedures dictated that a minimum of $5 \mathrm{~mm}$ of space must be maintained between the rack holding the specimens and the specimens themselves. This ensured that pooling of condensation off of the specimens did not exist at the bottom of the specimens and runoff from other specimens was not shared. Furthermore, the separate specimens, or assemblies of specimens were not allowed to contact one another, in order to prevent galvanic corrosion. 


\section{SALT SOLUTION APPLICATION}

After applicable accelerated corrosion studies were reviewed, it was decided that the most appropriate testing procedure for this study was the ASTM G44. In these studies, the ASTM G44[8] testing procedure was found to produce corrosion levels and products more consistent with in-field conditions as compared to the original procedure and was shown to be a viable alternative to in-field corrosion testing. With this in mind, the current research study implemented this accelerated cyclic corrosion test using a manual testing procedure.

The second stage in the corrosion testing procedure was the salt solution application stage, characterized by the exposure of the specimens to a highly corrosive salt solution for a period of 15 minutes in each cycle. The testing specification required this stage to be completed using one of three methods: the immersion method, the spray method, or the air atomized fog method. This stage was constrained to be completed using the immersion method. The process entailed full submersion of the corrosion specimens in a container filled with a solution characterized by a deionized reagent water solvent according to ASTM G44[8] and solutes with concentrations by mass: $5.0 \%$ sodium chloride $(\mathrm{NaCl})$. This process was completed at ambient temperatures, and thus, required no additional heating or cooling.

The preparation of this solution required that purified water be mixed with the aforementioned solute in a specific method and poured into the soaking containers to be used in this stage. Along with the physical preparation of the salt solution, records of the $\mathrm{pH}$ and conductivity of the solution were recorded whenever the solution was replaced.
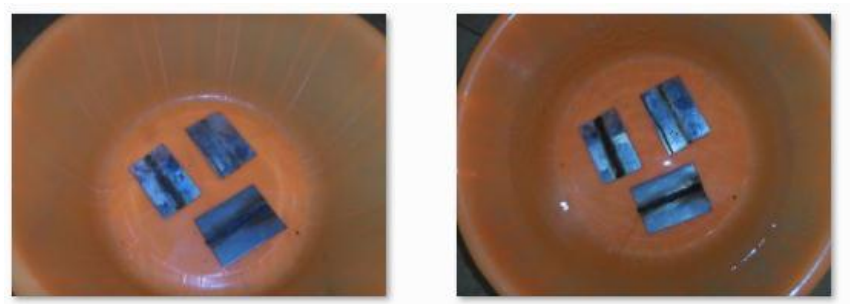

Fig 6: Immersion test

Within this step, the most time-consuming process was the purification of unpurified tap water to meet the requirements of the ASTM G44 specification. Based on recommendations in the testing specification, the salt solution was replaced on a weekly basis.

In order to facilitate the efficient and effective transfer of the racks containing the specimens back and forth from the environmental exposure to the soaking containers, noncorroding racks were used. The transfer process was completed manually, where racks were lifted in and out of the tubs by hand. When necessary, adjustments were made to the positioning of the specimens on the racks in order to ensure that specimens did not contact one another and were in the correct positioning. The fifteen-minute duration of the stage was ensured through timing of the stage during each day of testing.

\section{DRY STAGE}

The final stage of the corrosion cycle, the dry stage, was also completed. The duration of the stage was specified to be 17 hours and 45 minutes of continuous exposure, including the ramp step required to achieve the transition from the preceding conditions. At the completion of this stage, a 24-hour cycle was completed, to begin the ramp step of the next cycle within the humid stage.

\section{REAGENT WATER}

During various aspects of the accelerated testing procedure, including the preparation of the salt solution and steel cleaning solution, reagent water was required. For all instances, the deionized reagent water was prepared according to the prescription of ASTM G44.

As a result, the purification of the water was immediately verified, and the water was used to create the necessary solutions. Over the course of testing, a large demand of over 70 gallons per week was placed. The records which verify that acceptable conductivity and $\mathrm{pH}$ levels, as specified in ASTM $\mathrm{G} 44$, were achieved over the testing duration.

\section{TESTING SCHEDULE}

During the 120-cycle corrosion testing procedure, a number of activities were performed on a repeated basis. The order and schedule of these events will be described in detail in this section. Prior to testing, the specimens were prepared in order to ensure the removal of contamination from the specimens' surfaces. After preparation procedures had been completed, specimens were placed within the containers

The daily testing cycle involved the execution of the salt dip stage from 10:00 a.m. to 10:15 a.m., and dry stage from 10:15 a.m. to 8:00 a.m.; the following day. Every 10 day, pictures of representative samples from the specimens were taken. This process was facilitated by removing the racks and placing them on a table within the testing room. After specimens had been photographed, which lasted approximately 15 minutes, the racks were transferred into the soaking containers to begin the 15-minute salt application stage.

The testing procedure required the maintenance of equipment and the changing of salt solutions to occur on a regular basis. After the first week of testing, a routine was added to clean the containers to prevent further corrosion products, and refilling the containers back to the maximum fill line. Per the recommendations in the ASTM G44 specification, the salt solution was also replaced on a weekly basis.

In order to evaluate the corrosion behavior of the specimens, data measurements were also scheduled to be taken at intervals of 10 days. Data was collected at time periods of 40,80 , and 120 completed cycles.

\section{RESULT AND DISCUSSION}

In this section, the corrosion behavior data is presented in the form of average mass loss. This is a commonly used format for reporting corrosion testing data and is intended to provide the 
reader with a tangible physical representation of corrosion magnitudes experienced by the specimens. In these instances, the behavior and distribution of the mass loss is described. After the observational periods of 40, 80 and 120 cycles, The Percentage weight loss in the stainless steel welded using MMA, MIG, and TIG from the alternate immersion test was found to be $0.12 \%, 0.09 \%$ and $0.03 \%$, respectively.

Corrosion rate was calculated assuming uniform corrosion over the entire surface of the specimen. The corrosion rate in millimeter per year $(\mathrm{mm} / \mathrm{y})$ was calculated from the weight loss using the formula:

$$
\operatorname{CR}(\mathrm{mm} / \mathrm{y})=87.6 \times(\mathrm{w} / \mathrm{\rho At})
$$

Where;

w: weight loss in milligrams

$\rho:$ metal density in $\mathrm{g} / \mathrm{cm} 3$

A: area of sample in $\mathrm{cm} 2$

$\mathrm{t}$ : time of exposure of the metal sample in hours

Table 3. Weight in (g) after 40;80;120 cycles

\begin{tabular}{|c|c|c|c|c|}
\hline $\begin{array}{c}\text { Weight } \\
\text { loss }(\mathrm{g})\end{array}$ & $\begin{array}{c}0 \\
\text { cycles }\end{array}$ & 40 cycles & 80 cycles & $\begin{array}{c}120 \\
\text { cycles }\end{array}$ \\
\hline MMA & 108.1 & 108.06 & 108.02 & 107.97 \\
\hline MIG & 114 & 113.96 & 113.93 & 113.89 \\
\hline TIG & 110.6 & 110.59 & 110.58 & 110.56 \\
\hline
\end{tabular}

Table 4. Summary of the results

\begin{tabular}{|c|c|c|c|}
\hline & $\begin{array}{c}\text { Total weight } \\
\text { Loss }(\mathrm{g})\end{array}$ & $\begin{array}{c}\text { Percentage } \\
\text { weight loss } \\
(\%)\end{array}$ & $\begin{array}{c}\text { Corrosion } \\
\text { Rate CR } \\
(\mathrm{mm} / \mathrm{y})\end{array}$ \\
\hline MMA & 0.13 & 0.12 & 0.01 \\
\hline MIG & 0.11 & 0.09 & 0.01 \\
\hline TIG & 0.04 & 0.03 & 0.004 \\
\hline
\end{tabular}

The Corrosion rates after 120 cycles were similar for MMA, MIG equal to $0.01 \mathrm{~mm} / \mathrm{y}$, and $0.004 \mathrm{~mm} / \mathrm{y}$ for TIG, giving an average of weight loss $0.12 \% ; 0.09 \%$ and $0.03 \%$ respectively.

These results showed a general pattern of increase in percentage weight loss (PWL) and a corresponding increase in corrosion rate $(\mathrm{CR})$ in the samples, with respect to time. An inverse proportional trend seems to exist between the weight loss and corrosion rate over time.

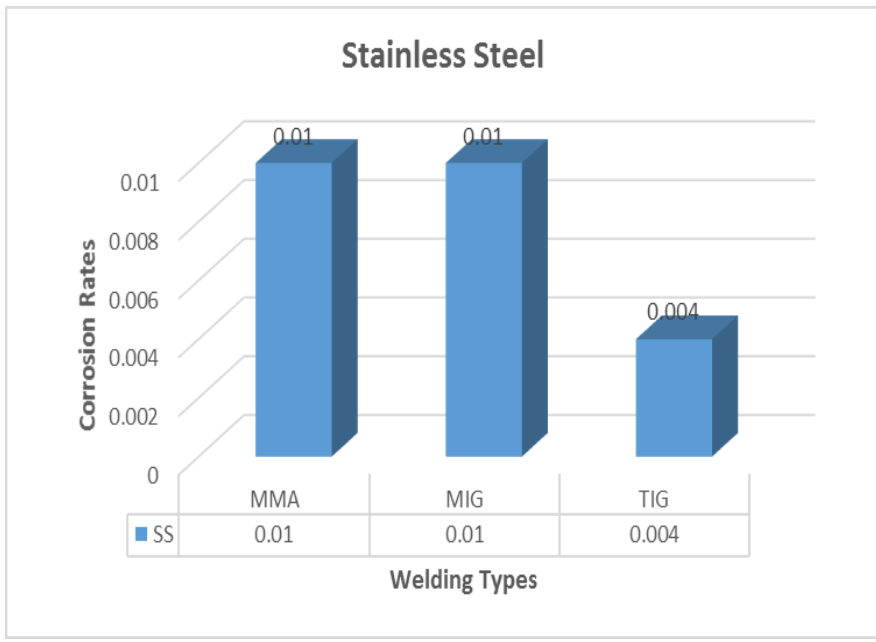

Fig 7: Corrosion rate of stainless steel welded using MMA, MIG, and TIG

The objective of testing specimens was the collection of baseline data of mass loss and corrosion behavior for stainless steel being considered in testing. This ultimately allowed for comparisons with plates in the other studies and with previous corrosion studies on metal materials. Behavior observed for the specimens indicated that the main controlling factor determining the corrosion was the type of metal in consideration and the ability, or inability, of the metal to form a passive surface layer in the highly-corrosive testing environment. as well as the welding effect.

\section{HEAT TREATMENT}

The heat treatment was done to investigate the influence on the corrosion behavior of the material, The stainless steel is heated until $600^{\circ} \mathrm{C}$ temperature and then air-cooled. The normalized specimens have improved hardness, strength and machinability. This heat treatment is more extensively used since it is more economical.

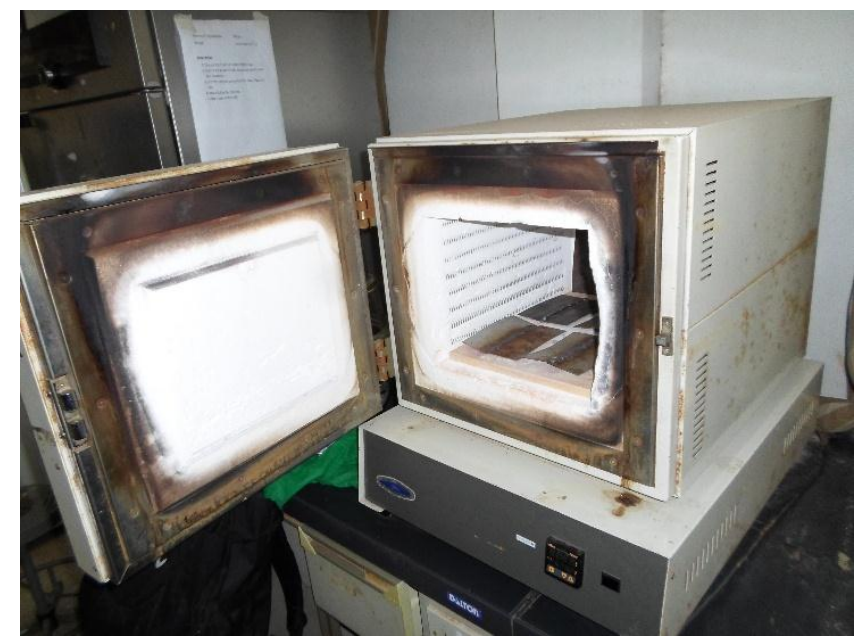

Fig 8: Heat Treatment of Stainless steel 
Table 5: Summary of the results

\begin{tabular}{|c|c|c|c|}
\hline & $\begin{array}{c}\text { Total Weight } \\
\text { Loss in gram }\end{array}$ & $\begin{array}{c}\text { Percentage } \\
\text { weight loss } \\
(\%)\end{array}$ & $\begin{array}{c}\text { Corrosion } \\
\text { Rate CR } \\
(\mathrm{mm} / \mathrm{y})\end{array}$ \\
\hline MMA & 0.09 & 0.08 & 0.004 \\
\hline MIG & 0.05 & 0.03 & 0.003 \\
\hline TIG & 0.01 & 0.01 & 0.0001 \\
\hline
\end{tabular}

Corrosion rates of the specimens showed decreasing trend before it remained consistent throughout. The rate of corrosion for all specimens was low because of the change in the physical properties of the metal. Normalized stainless steels are harder and stronger than normal stainless steel that explain their corrosion resistance.

\section{CONCLUSION}

This study compares the corrosion resistance of MMA, MIG and TIG under atmospheric exposures and in accelerated tests. Through the image analysis and the alternate immersion tests, it has been observed that the TIG welds have the highest corrosion resistance in atmospheric exposures. MIG and welds have the least corrosion resistance. And they corrode as rapidly as the base metal.

Arc welding and MIG are very common welding techniques used in industry. Although it has some essential advantages for its extensive use, including its ease of use and the quality of the weld, if used in a corrosive media without appropriate corrosion prevention methods, it may be subject to high corrosion rates which may lead to serious corrosion problems.

The study shows the effect of exposure time on weight loss and corrosion resistance of the material and the effects of the heat treatment on the stainless steel, It shows an increase in the corrosion resistance of the material due mainly to normalizing process.

\section{REFERENCES}

[1] Surnam, B. Y. R., et al. "Long term atmospheric corrosion in Mauritius." Corrosion Engineering, Science and Technology 50.2 (2015): 155-159.

[2] ISO, EN. "9226: Corrosion of metals and alloys." Corrosivity of atmospheres. Determination of corrosion rate of standard specimens for the evaluation of corrosivity.(ISO 9226: 2012) (2012).

[3] Surnam, B. Yashwansingh R., and Yashwantraj Seechurn. "Pitting corrosion of aluminium in the tropical climate of Mauritius." Corrosion Engineering, Science and Technology 52.6 (2017): 411-417.Beeharry, P., and B. Y. R. Surnam. "Atmospheric corrosion of welded mild steel." Materials Today: Proceedings 5.2 (2018): 74767485.

[4] Ban, Hui Yong, et al. "Research progress on the mechanical property of high strength structural steels." Advanced Materials Research. Vol. 250. Trans Tech Publications, 2011.

[5] Standard, A. S. T. M. "Standard practice for preparing, cleaning, and evaluating corrosion test specimens." American Society for Testing and Materials G1-03 (2011).

[6] Perić, Mato, et al. "Numerical analysis and experimental investigation of welding residual stresses and distortions in a T-joint fillet weld." Materials \& Design 53 (2014): 1052-1063.

[7] ISO 15614-1, Specification and qualification of welding procedures for metallic materials -- Welding procedure test -- Part 1: Arc and gas welding of steels and arc welding of nickel and nickel alloys, 2004.

[8] ASTM G44, Standard practice for exposure of metals and alloys by alternate immersion in neutral $3.5 \%$ Sodium Chloride solution, 2004.

[9] ASTM G 31, Standard Practice for Laboratory Immersion Corrosion Testing of Metals, 2004

[10] ASTM E562-11, Standard Test Method for Determining Volume Fraction by Systematic Manual Point Count, 2011.

[11] ISO, BSEN. "8565, Metals and alloys: atmospheric corrosion testing." General requirements (1995). 